\title{
ARTICLE \\ Evidence for interplay among antibacterial-induced gut microbiota disturbance, neuro-inflammation, and anxiety in mice
}

\author{
Hyo-Min Jang ${ }^{1}$, Hae-Ji Lee ${ }^{1}$, Se-Eun Jang ${ }^{1,2}$, Myung Joo Han ${ }^{2}$ and Dong-Hyun Kim ${ }^{1}$
}

\begin{abstract}
The aim of the present study was to determine whether there is the mechanistic connection between antibacterial-dependent gut microbiota disturbance and anxiety. First, exposure of mice to ampicillin caused anxiety and colitis and increased the population of Proteobacteria, particularly Klebsiella oxytoca, in gut microbiota and fecal and blood lipopolysaccharide levels, while decreasing lactobacilli population including Lactobacillus reuteri. Next, treatments with fecal microbiota of ampicillin-treated mouse (FAP), $K$. oxytoca, or lipopolysaccharide isolated from $K$. oxytoca $(\mathrm{KL})$ induced anxiety and colitis in mice and increased blood corticosterone, IL-6, and lipopolysaccharide levels. Moreover, these treatments also increased the recruitment of microglia (Iba $1^{+}$), monocytes $\left(\mathrm{CD} 11 \mathrm{~b}^{+} / \mathrm{CD} 45^{+}\right)$, and dendritic cells $\left(\mathrm{CD} 11 \mathrm{~b}^{+} / \mathrm{CD} 11 \mathrm{c}^{+}\right)$to the hippocampus, as well as the population of apoptotic neuron cells (caspase- $3^{+} / \mathrm{NeuN}^{+}$) in the brain. Furthermore, ampicillin, $K$. oxytoca, and KL induced NF-KB activation and IL- $1 \beta$ and TNF- $\alpha$ expression in the colon and brain as well as increased gut membrane permeability. Finally, oral administration of $L$. reuteri alleviated ampicillin-induced anxiety and colitis. These results suggest that ampicillin exposure can cause anxiety through neuroinflammation which can be induced by monocyte/macrophage-activated gastrointestinal inflammation and elevated Proteobacteria population including $K$. oxytoca, while treatment with lactobacilli suppresses it.
\end{abstract}

Mucosal Immunology (2018) 11:1386-1397; https://doi.org/10.1038/s41385-018-0042-3

\section{INTRODUCTION}

Anxiety disorders are the most common psychiatric illness. ${ }^{1}$ Approximately one-eighth of the world's population suffers from them. ${ }^{2,3}$ Anxiety-like behaviors are approximated in experimental animals by exposure to various stressors, such as immobilization, forced swimming, and social defeat. ${ }^{4}$ Stressors stimulate the secretion of glucocorticoids, adrenaline, and noradrenaline through the hypothalamo-pituitary-adrenal axis, increase the expression and secretion of proinflammatory cytokines in immune cells, ${ }^{5-9}$ and disturb the composition of gut microbiota. $^{10,} 11$ Gut microbiota have been implicated in the development and functioning of several basic physiological processes, including homeostasis maintenance, and play a role in multiple chronic diseases, such as obesity, chronic inflammatory diseases, and asthma. ${ }^{12}$ Gut microbiota and their byproducts can stimulate the immune and central nervous systems (CNS). ${ }^{11}$, 13 These complex interactions are considered to form a microbiota-gut-brain axis regulated through the CNS; enteric nervous system (ENS); and neural, endocrine, and immune systems. ${ }^{14,15}$ Gut microbiota disturbance has been continuously reported to be associated with the occurrence of neuropsychiatric disorders such as schizophrenia, autistic disorders, and anxiety and depressive disorders, ${ }^{16}, 17$ as well as gastrointestinal (GI) inflammatory diseases such as colitis. ${ }^{18,19}$ However, the effects of antibacterials, gut microbiota disrupters, against anxiety are contradictory. $^{20-24}$ Leclercq et al. $^{22}$ reported that early-life antibiotic exposure decreased anxiety-like behavior in male mice, while Atli et al. ${ }^{21}$ reported that amoxicillin exposure increased depression in rats. Moreover, some anti-bacterials disturb gut microbiota composition, resulting in $\mathrm{Gl}$ inflammation. ${ }^{25}$ Therefore, the clear mechanism of antibacterials on the occurrence of anxiety remains elusive.

Microbial colonization in the Gl tract of germ-free mice is central to the development and maturation of both ENS and CNS. ${ }^{26,} 27$ This is associated with the expression and turnover of neurotransmitters in the nervous systems $\mathrm{s}^{26,28,29}$ and cytokines in the Gl tract. ${ }^{30}$ Particularly, germ-free mice display reduced anxiety-like behaviors in the elevated plus maze (EPM) task compared to specific pathogen-free (SPF) mice and increased brain-derived neurotrophic factor (BDNF) expression in the dorsal hippocampus. ${ }^{29,} 31$ Exposure to stressors such as social defeat and food and water deprivation in SPF animals causes anxiety, increases cortisol levels, decreases the abundance of beneficial bacteria, and increases pathogen susceptibility in mice. ${ }^{32-35}$ Furthermore, these stressors deplete Lactobacillus species like Lactobacillus reuteri. ${ }^{36}$ However, oral administration of some probiotics such as L. reuteri, which can restore the disturbed gut microbiota composition, induces anxiolytic effect in mice. ${ }^{17,} 37$ These findings suggest that maintaining the gut microbiota balance plays an important role in the occurrence of anxiety.

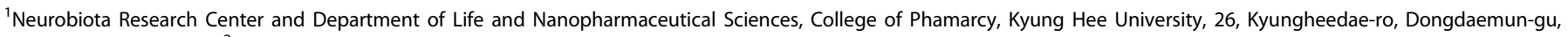
Seoul 02447, Korea and ${ }^{2}$ Department of Food and Nutrition, Kyung Hee University, 26, Kyungheedae-ro Dongdaemun-gu, Seoul 02447, Korea

Correspondence: D-H. Kim (dhkim@khu.ac.kr)

These authors contributed equally: Hyo-Min Jang, Hae-Ji Lee

Received: 28 December 2017 Revised: 21 April 2018 Accepted: 29 April 2018

Published online: 4 June 2018 
Therefore, to understand whether antibacterial-induced gut microbiota disturbance could cause anxiety and what kinds of bacteria could be targeted to accelerate or alleviate anxiety, we examined the effect of ampicillin, a gut microbiota-disturbing antibacterial, on the composition of gut microbiota, the occurrence of anxiety, and neuro-inflammation markers in mice. The data presented in this study support a mechanistic link between gut microbiota change and anxiety potentially through endotoxinmediated neuro-inflammation.

\section{RESULTS}

Ampicillin caused anxiety and colitis in mice

First, to understand whether the gut microbiota composition disturbance by antibacterials could affect anxiety, we orally administered ampicillin to mice once a day for 2 days and measured anxiety-like behaviors on the 4th and 10th day after the final treatment with ampicillin (Fig. 1a and supplement Figure S1 and S2). Exposure of mice to ampicillin dose-dependently decreased the time spent in open arms (OT) and open arm entries (OE) in the EPM task. When ampicillin at a dose of $100 \mathrm{mg} /$ $\mathrm{kg}$, which is equivalently $500 \mathrm{mg} / 60 \mathrm{~kg} /$ day in human dose (based on the $\mathrm{Km}$ factor Guidance for the industry from the Food and Drug Administration (FDA)), was orally administered, OT and OE reached to the lowest levels (Supplement Figure S1). Ampicillin treatment also increased anxiety-like behaviors in the light/dark transition (LDT) and marble-burying (MB) tasks (Fig. 1b-d). Moreover, ampicillin treatment suppressed BDNF expression and increased NF-KB activation in the hippocampus. Ampicillin treatment also significantly increased corticosterone, interleukin6 (IL-6), tumor necrosis factor-a (TNF-a), and lipopolysaccharide (LPS) levels in the blood (Fig. 1e, f and Supplement Figure S1). Furthermore, ampicillin treatment significantly increased the populations of microglia $\left(\mathrm{lba} 1^{+}\right)$, monocytes $\left(\mathrm{CD} 11 \mathrm{~b}^{+} \mathrm{CD} 45^{+}\right)$, dendritic cells (DCs, CD11 $\left.\mathrm{b}^{+} \mathrm{CD} 11 \mathrm{c}^{+}\right)$, and apoptotic neuron cells (caspase- $3^{+} / \mathrm{NeuN}^{+}$) and LPS levels in the brain including the hippocampus and cortex, as well as the expression of IL-1R and IL$1 \beta$ (Fig. $1 \mathrm{~g}-\mathrm{l}$ ), assessed by quantitative polymerase chain reaction (qPCR) and fluorescence-activated cell sorting. Ampicillin treatment increased brain LPS levels (Fig. $1 \mathrm{~m}$ ). Moreover, ampicillin treatment also induced myeloperoxidase activity that was not accompanied by colon shortening, as well as NF-KB activation and inducible nitric oxide synthase (iNOS), COX-2, IL-6, and TNF-a expression in the colon (Fig. $1 \mathrm{n}-\mathrm{q}$ and Supplement Figure S1). Monocytes and DCs were recruited in the colon by treatment with ampicillin, which also induced the expression of IL-1R, myeloperoxidase, and IL-1 $\beta$. However, treatment with ampicillin suppressed occludin, claudin-1, and IL-10 expression in the colon. Furthermore, ampicillin treatment increased the gut membrane permeability: it increased the absorption of orally administered fluorescent fluorescein isothiocyanate (FITC)-dextran into the blood (Fig. 1q). Ampicillin treatment significantly increased the population of Proteobacteria in the gut microbiota whereas the Actinobacteria population decreased (Supplement Figure S2). Furthermore, ampicillin treatment increased brain LPS levels (Fig. 1r).

Next, we orally administered ampicillin to mice, collected feces on the 10th day after treatment with ampicillin, orally administered the fecal microbiota of ampicillin-treated mice (FAP) or normal control mice (FNC), and measured the resultant anxiety-like behaviors on the 5th day after the final treatment of FAP or FNC (Fig. 2a-c and Supplement Figure S3). Treatment with FAP significantly increased anxiety-like behaviors, suppressed BDNF expression, and increased NF-KB activation in the hippocampus, which were similar to those in ampicillin-treated mice (Fig. 2d). FAP treatment increased blood corticosterone, IL-6, TNF-a, and LPS levels (Fig. 2e, f and supplement Figure S3). Exposure to FAP significantly increased microglia $\left(\mathrm{Iba} 1^{+}\right)$, monocytes $\left(\mathrm{CD} 11 \mathrm{~b}^{+} \mathrm{CD} 45^{+}\right)$, DCs $\left(\mathrm{CD} 11 \mathrm{~b}^{+} \mathrm{CD} 11 \mathrm{c}^{+}\right)$, and apoptotic neuron cells (caspase $-3^{+} / \mathrm{NeuN}^{+}$) populations and LPS levels in the hippocampus (Fig. 2g-k). FAP treatment increased brain LPS levels (Fig. 2I). FAP treatment also induced myeloperoxidase activity; NF-KB activation; and iNOS, COX-2, TNF-a, and IL-6 expression in the colon (Fig. $2 \mathrm{~m}-\mathrm{o}$ ). FAP treatment also recruited monocytes and DCs in the colon (Fig. 2q). Furthermore, treatment with FAP suppressed occludin, claudin-1, and IL-10 expression. However, FNC treatment did not affect anxiety and hippocampal BDNF expression and NF-KB activation, as observed in the normal control mice treated with vehicle only. Treatment with FAP significantly increased the Proteobacteria population and decreased the Actinobacteria population while those in FNC-treatment mice were not significantly different to those in normal control mice (Supplement Figure S3). FAP treatment also increased fecal LPS levels (Fig. 2p).

Ampicillin increased the Proteobacteria population in mice Exposure to ampicillin caused anxiety as well as disturbed gut microbiota composition, assessed by qPCR. The gavage of FAP also caused anxiety and gut microbiota disturbance. Therefore, to identify the commensal gut bacteria that could induce anxiety in vivo, first we first investigated the difference in gut microbiota composition between mice treated or not treated with ampicillin by culturing in the selective media (Fig. 3a). Treatment with ampicillin increased the abundance of Klebsiella oxytoca, Escherichia coli, and Morganella morganii, which belong to Proteobacteria, and Aerococcus urinaeequi, which belongs to Firmicutes, whereas the abundances of Lactobacilli, including $L$. reuteri, Lactobacillus johnsonii, and Lactobacillus plantarum, were reduced. Of these bacteria, $K$. oxytoca most potently increased anxiety-like behaviors in the EPM task, followed by E. coli (Supplement Figure S4).

Therefore, to confirm the disturbance of gut microbiota composition, we analyzed fecal microbiota by pyrosequencing (Fig. 3b-d). The bacterial richness and diversity in mice treated with ampicillin or $K$. oxytoca were significantly lower than in normal control mice, as demonstrated by the number of sequences analyzed, estimated operational taxonomic unit (OTU) richness, and coverage. However, the bacterial richness and diversity between mice treated with FNC was not significantly different. Comparing the results of taxonomy-based analysis between mice treated and not treated with ampicillin, FAP, or $K$. oxytoca, it revealed that treatment with ampicillin or $K$. oxytoca induced a significant disturbance of the populations. At the phylum level, treatment with ampicillin led to a significant increase in the population of Proteobacteria and Bacteroidetes. Moreover, treatment with ampicillin, FAP, or $K$. oxytoca increased the proportion of Bacteroidetes and Proteobacteria and reduced the proportion of Firmicutes. However, treatment with FNC did not significantly affect the proportion of Proteobacteria and Bacteroidetes. At the family level, treatment with ampicillin, FAP, or $K$. oxytoca significantly increased the proportions of Prevotellaceae, Enterobacteriaceae, and Desulfovibrionaceae but reduced the proportions of Lactobacillaceae. Furthermore, ampicillin treatment reduced the proportions of Ruminococaceae, Rikenellaceae, and Lachnospiraceae. FAP treatment reduced the proportions of Ruminococaceae. K. oxytoca treatment reduced the proportion of Lachnospiraceae and Rikenellaceae. At the genus level, treatment with ampicillin or $K$. oxytoca increased the proportions of Klebsiella sp. and Prevotellaceae_uc sp. Furthermore, ampicillin treatment increased the proportions of Bacteriodes sp. and EF406806_g sp.

Next, we processed these sequences to match the length and position of the gut microbiota 16S rRNA gene sequences; computed all pair-wise distances among vehicle alone, ampicillin-, FAP-, or K. oxytoca-treated mice; and performed principal 
H-M Jang et al.
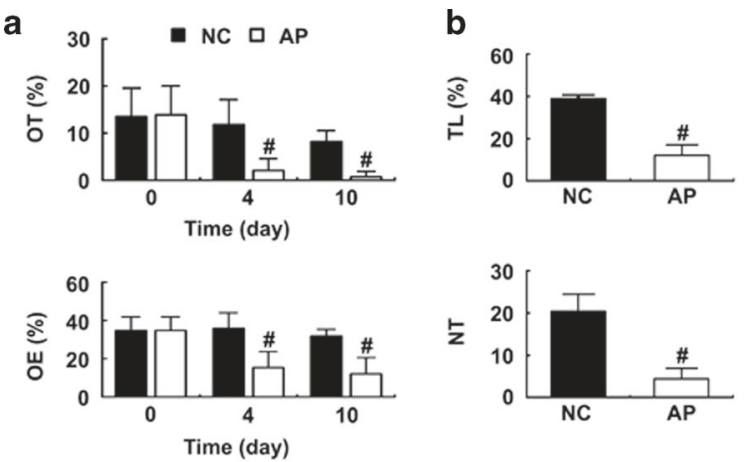

C

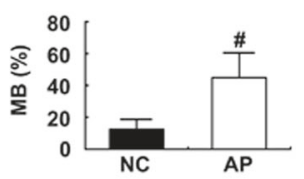

d
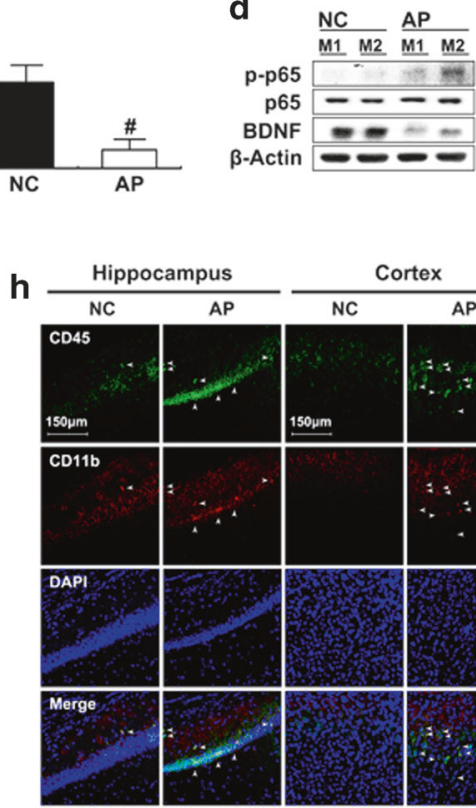

$\mathbf{k}$
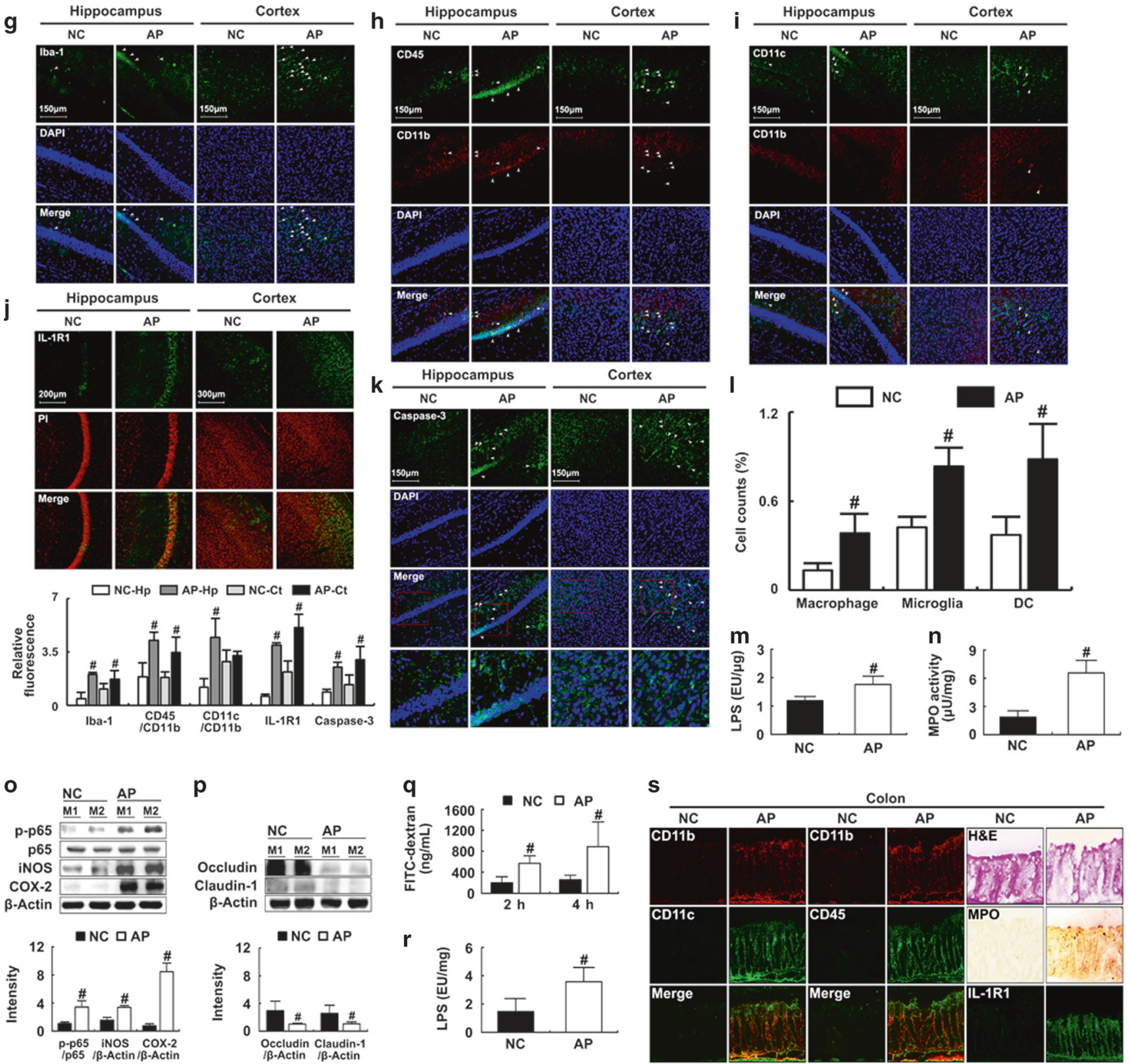

coordinate analysis (Fig. 3c). The gut microbial community of normal control mice differed significantly from those of mice treated with ampicillin, FAP, or K. oxytoca, while the gut microbial community of FNC-treated mice did not significantly differ from that of normal control mice.
$K$. oxytoca induced anxiety and colitis in mice

To understand whether $K$. oxytoca could cause anxiety through gut inflammation, we investigated the effect of $K$. oxytoca on anxiety-like behaviors and colitis marker expression in mice (Fig. 4 and Supplement Figure S5). K. oxytoca treatment increased 
Fig. 1 Ampicillin caused anxiety-like behaviors and colitis in mice. Anxiety-like behaviors of mice were measured in the EPM (a), LDT (b), and $M B$ tests (c). EPM task (time spent in open arms (OT) and open arm entries (OE)) was measured on the 4th and 10th day after the initial ampicillin treatment and LDT and MB tasks were on 10th day. Normal control mice (NC) were treated with vehicle alone. NF- $\mathrm{kB}$, BDNF, and $\beta$ actin were measured in the hippocampi by immunoblotting (d). Blood corticosterone (e) and LPS levels (f) were analyzed by ELISA or LAL kit. Microglia (g), monocytes (h), and dendritic cells (i) recruited/activated into the brain, IL-1R expression (j) levels, and apoptotic neuron cells (k) were assayed by a confocal microscope. Microglia, monocytes, and dendritic cells were also assayed by a flow cytometer (I). Brain LPS levels were measured by LAL assay kit $(\mathbf{m})$. Colitis markers myeloperoxidase activity (n) and iNOS, COX-2, NF- $\kappa B$ (o), and tight junction proteins (occludin and claudin-1), and $\beta$-actin (p) were assessed by immunoblotting. Orally administered FITC-dextran was absorbed into the blood (q). Fecal LPS levels ( $\mathbf{r}$ ) were measured by LAL assay kit. Macrophages, dendritic, and IL-1R1-expressing cells (s) recruited/activated into the colon were assayed by a confocal microscope. NC and AP in the $x$-axis of figures indicate groups treated with vehicle alone and ampicillin, respectively. All data were expressed as mean \pm SD $(n=8) ;{ }^{\#} p<0.05$ vs. normal control (NC) group

anxiety-like behaviors in LDT and MB tasks. The intraperitoneal injection of LPS purified from $K$. oxytoca (KL) also showed anxietylike behaviors (Fig. $4 \mathrm{~d}$ and supplement Figure S5). Furthermore, treatment with $K$. oxytoca or KL suppressed BDNF expression and increased NF-KB activation in the hippocampus (Fig. 4b, e). Moreover, $K$. oxytoca or $\mathrm{KL}$ treatment led to significantly higher blood LPS, corticosterone, IL-6, and TNF-a levels than those in the normal control group (Fig. 4c, f and supplement 5S). Furthermore, oral gavage of $K$. oxytoca or $\mathrm{KL}$ significantly increased the populations of microglia, monocytes, and DCs in the brain as well as the expression of IL-1R and IL-1 $\beta$ (Fig. $4 \mathrm{~g}-\mathrm{I}$ ), which are typically observed during neuro-inflammation. ${ }^{38} \mathrm{~K}$. oxytoca or $\mathrm{KL}$ treatment also increased apoptotic neuron cells in the brain. Moreover, exposure to $K$. oxytoca or $\mathrm{KL}$ induced colon shortening and myeloperoxidase activity. $K$. oxytoca or $\mathrm{KL}$ treatment also upregulated NF-KB activation and iNOS, COX-2, TNF- $\mathrm{a}$, and IL-6 expression (Fig. 4p). Moreover, $K$. oxytoca or $\mathrm{KL}$ treatment significantly recruited monocytes and DCs in the colon and induced the expression of IL-1R, myeloperoxidase, and IL-1 $\beta$ (Fig. 4t). However, K. oxytoca suppressed occludin, claudin-1, and IL-10 expression in the colon (Fig. 4q). K. oxytoca treatment increased the Proteobacteria population (Supplement Figure S5) and fecal LPS levels (Fig. 4s). Moreover, K. oxytoca treatment increased the absorption of orally administered FITC-dextran into the blood (Fig. 4r). However, intraperitoneal injection of L. reuteri exopolysaccharide in mice did not induce anxiety-like behaviors in EPM task and colitis markers such as colon shortening and myeloperoxidase activity (Supplement Figure S6).

Lactobacilli alleviated anxiety and colitis in mice

Next, we investigated the effects of $L$. reuteri, $L$. johnsonii, $L$. plantarum, and Lactobacillus rhamnosus, which were significantly reduced by treatment with ampicillin, on ampicillin-induced anxiety and colitis in mice (Fig. 5a and Supplement Figure S7). Ampicillin exposure significantly increased anxiety-like behaviors in the EPM task. However, these lactobacilli $\left(1 \times 10^{9}\right.$ colonyforming units (CFUs)/mouse) inhibited ampicillin-induced anxiety. Of the tested lactobacilli, L. reuteri most potently attenuated ampicillin-induced anxiety. $L$. reuteri treatment increased ampicillin-suppressed BDNF expression and inhibited ampicillininduced NF-KB activation in the hippocampus. Furthermore, treatment with $L$. reuteri significantly inhibited ampicillin-induced blood levels of corticosterone, IL-6, and TNF-a. L. reuteri significantly alleviated ampicillin-induced colitis: $L$. reuteri reduced myeloperoxidase activity; NF-KB activation; and TNF-a, IL-6, iNOS, and COX-2 expression. Furthermore, $L$. reuteri attenuated $K$. oxytoca-induced anxiety-like behaviors and colitis. Moreover, $L$. reuteri alleviated $K$. oxytoca-induced anxiety and colitis in mice (Fig. 5b and Supplement Figure S7).

\section{DISCUSSION}

Recently, a series of studies have highlighted the influence of the gut microbiota on neuropsychiatric disorders (via the microbiota-gut-brain axis). ${ }^{39}, 40$ Exposure of mice to stressors decreased the abundance of Bacteroides sp. and Clostridium sp. in the gut microbiota and led to higher levels of inflammatory cytokines and neuroendocrine hormones such as IL-6 and corticosterone than in non-stressed control mice. ${ }^{34,41}$ However, exposure of germ-free or antibiotic-treated mice to stressors led to greater motor activity and lower anxiety than that in SPF mice with normal gut microbiota. ${ }^{29}$ Germ-free mice with transplanted fecal microbiota (bacteria) from SPF mice demonstrated a removal of the reduced anxiety in germ-free mice. ${ }^{28,29}$ In contrast, Lurie et al. ${ }^{24}$ reported that exposure to antibiotics is associated with increased risk for depression and anxiety. ${ }^{24}$ Fröhlich et al. reported that oral gavage of an antibiotic mix (ampicillin, bacitracin, meropenem, neomycin, and vancomycin), which did not enter the brain, caused gut dysbiosis and memory impairment in novel object recognition task. ${ }^{42}$ Desbonnet et al. ${ }^{41}$ reported that treatment with antibiotic mixture (ampicillin, vancomycin, neomycin, metronidazole, and amphotericin-B) impaired gut microbiota composition and anxiety and cognitive function in mice, which are similar to that reported in germ-free mice. Hoban et al. ${ }^{43}$ reported that the depletion of gut microbiota by other antibiotic mixture (ampicillin, vancomycin, ciprofloxacin, imipenem, and metronidazole) caused memory deficits in spatial memory depressive-like behaviors. Vogt et al. ${ }^{44}$ reported that antibiotic minocycline, even though intraperitoneally injected, did not display antidepressant- or anxiolytic-like effects of diazepam in mice. These findings indicate that the GI microbiota alteration in mice can result in measurable changes in anxiety.

The microbiota-gut-brain axis connecting the Gl tract and CNS is regulated through neuronal, endocrinal, and immunological signaling. $5,7,8,10$ The secretion of neuroendocrine hormones (e.g., cortisol) by stressors alters gut permeability and barrier function and facilitates communication with immune cells, which regulate defense responses to pathogen infection by the release of cytokines such as TNF- $a$ and IL-6, leading to the occurrence of immune disorders. ${ }^{39}$ Thus, dysfunction of this gut-brain axis can cause inflammation, anxiety, and depression.

In the present study, we also found that exposure to ampicillin caused anxiety-like behaviors and disturbed gut microbiota; ampicillin increased the population of Proteobacteria, particularly $K$. oxytoca and $E$. coli belonging to $\gamma / \delta$-Proteobacteria. These results suggest that the disturbed gut microbiota composition in ampicillin-treated mice cannot be easily restored to those of normal control mice. Furthermore, ampicillin treatment increased gut microbiota LPS levels in colonic fluids and blood. These results were supported by the analysis of gut microbiota using pyrosequencing. Treatment with ampicillin deteriorated the expression of colitis markers such as myeloperoxidase activity, TNF-a expression, and tight junction proteins and increased the activation of NF-KB in the colon: these caused colitis. Ampicillin treatment also increased the gut membrane permeability to FITCdextran. However, live bacteria were not detected in the blood and liver by using GAM and $\mathrm{DHL}$ agar plates (data not shown). Moreover, we also found that ampicillin treatment reduced BDNF expression in the hippocampus and increased NF-KB activation in the hippocampus and colon. Ampicillin treatment increased the 
H-M Jang et al.

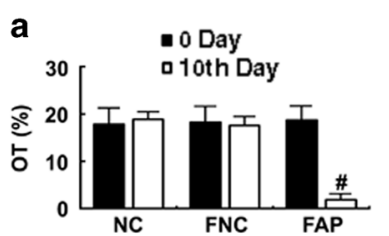

b
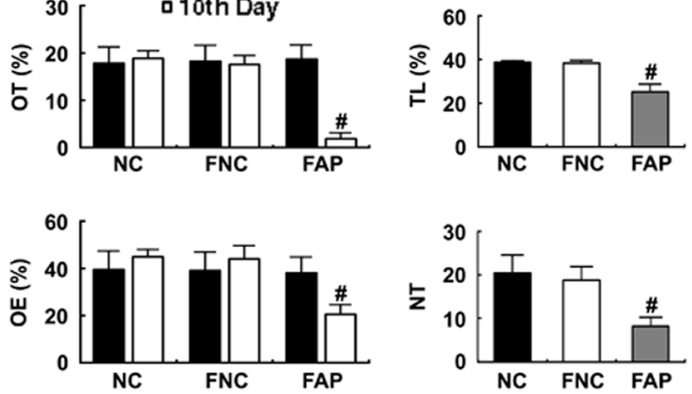

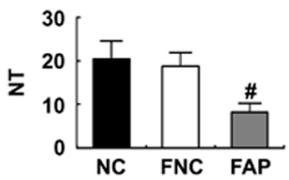

c

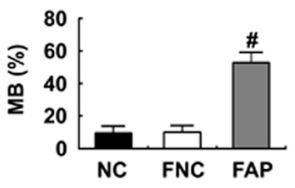

d

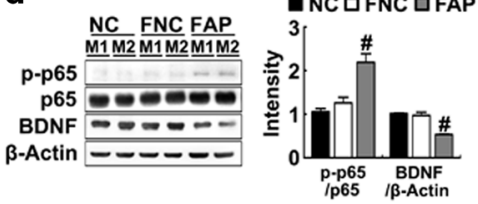

e
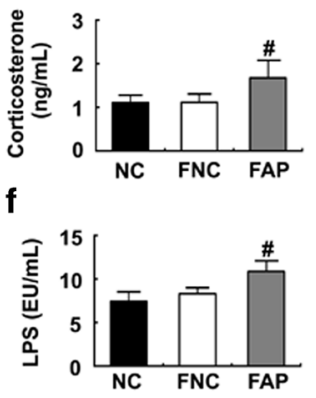

g
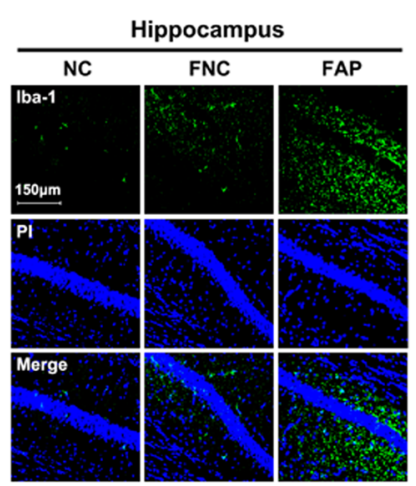

j
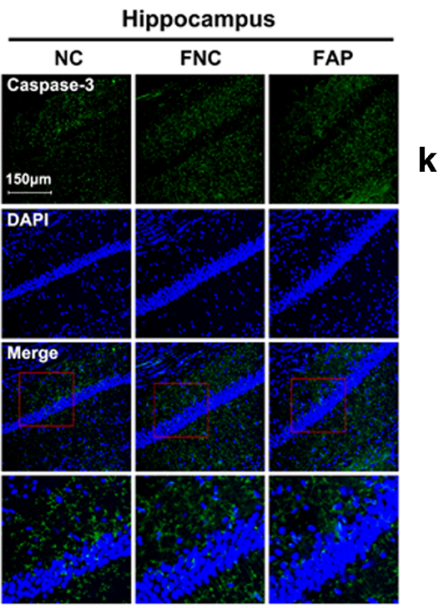

I

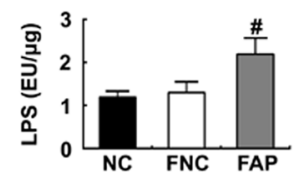

m

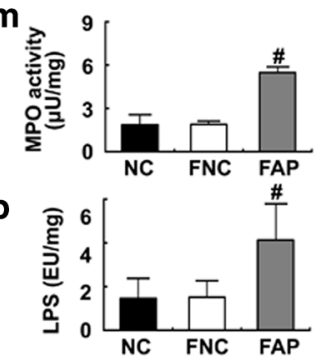

\section{q}

h

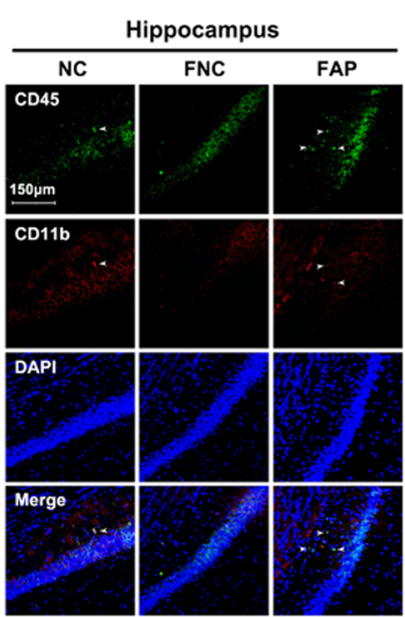

$\mathbf{k}$

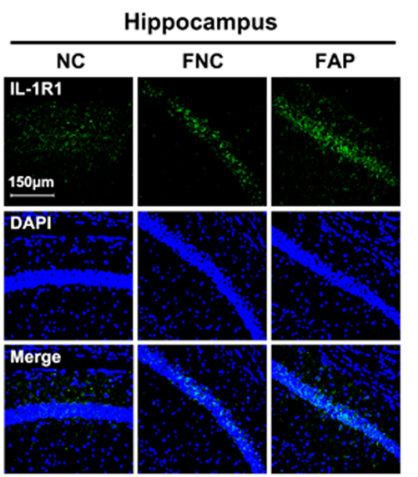

i

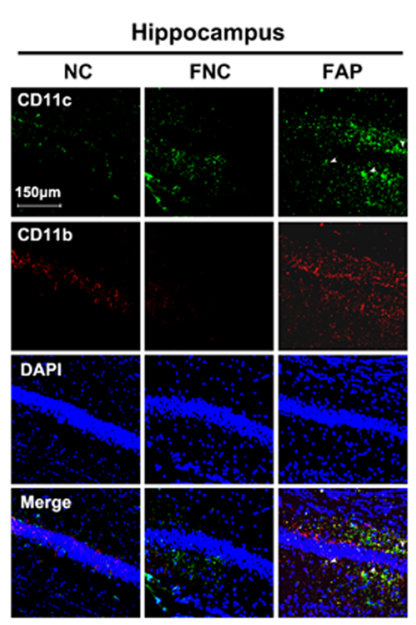

n

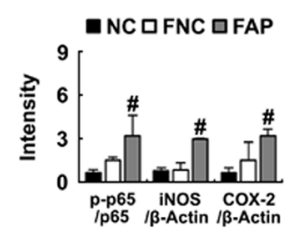

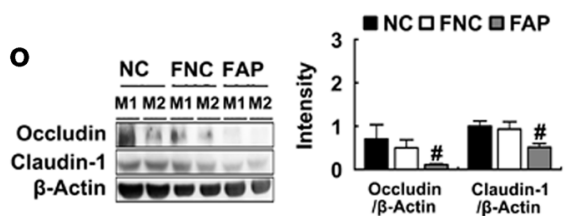

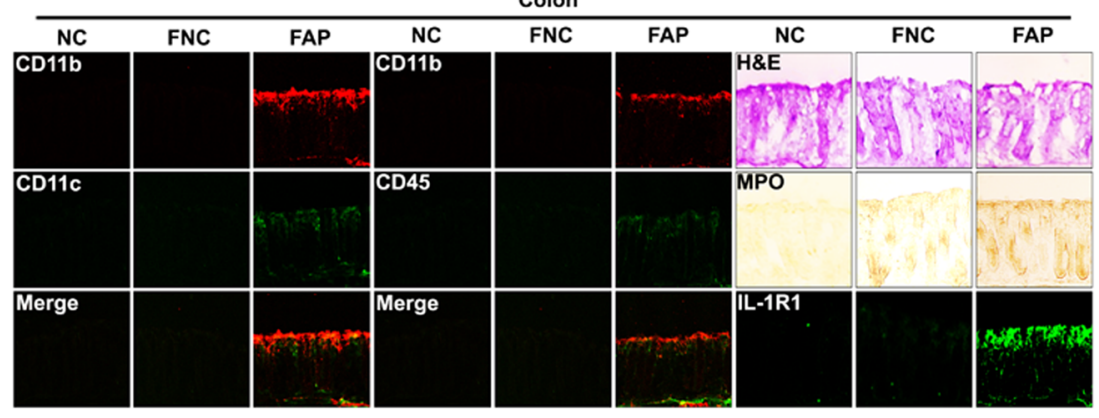

recruits of microglia, DC, and monocytes and induced IL-1R and IL$1 \beta$ expression in the brain. Additionally, stress-induced microglia induced the population of IL-1 $\beta$-expressing monocytes through IL$1 \mathrm{R}$ expression. ${ }^{38}$ We found that ampicillin treatment also increased apoptotic neuron cells. These results suggest that ampicillin can cause colitis by gut microbiota disturbance and LPS increment, then increase blood LPS levels, and activate blood monocytes. The activated monocytes are delivered to the brain and induce the 
Fig. 2 Fecal microbiota from ampicillin-treated mice (FAP) induced anxiety-like behaviors and colitis in mice. Anxiety-like behaviors of mice were measured in the EPM (a), LDT (b), and MB tests (c). EPM, LDT, and MB tasks were on the 5th day after the final gavage of FAP. Effect in the NF-KB activation and BDNF expression (d). Blood corticosterone (e) and LPS levels (f), were analyzed by ELISA or LAL kit. Microglia (g), monocytes (h), and dendritic cells (i), recruited/activated into the hippocampus, apoptotic neuron cells (j), and IL-1R1 expression (k), levels were assayed by a confocal microscope. Brain LPS levels were measured by LAL assay kit (I). Colitis markers myeloperoxidase activity (m) and iNOS, COX-2, NF-KB (n), and tight junction proteins (occludin and claudin-1), and $\beta$-actin (o), were assessed by immunoblotting. Fecal LPS levels (p), were measured by LAL assay kit. Macrophages, dendritic, and IL-1R1-expressing cells (q), recruited/activated into the colon were assayed by a confocal microscope. NC, FNC, and FAP in the $x$-axis of figures indicate groups treated with vehicle alone, the fecal microbiota of normal control mice, and the fecal microbiota of ampicillin-treated mice, respectively. All data were expressed as mean \pm SD $(n=8) ;{ }^{\#} p<0.05$ vs. normal control (NC) group

expression of IL-1 $\beta$ and IL-1R and activation of microglia, which may repeatedly activate IL-1 $\beta$-expressing monocytes and suppress BDNF expression, resulting in the occurrence of anxiety. Additionally, many endotoxins isolated from gut bacteria such as $E$. coli could activate monocytes and might deteriorate anxiety and cognitive function. ${ }^{14,18,19,36}$ However, Bercik and Collins ${ }^{23}$ have reported that oral administration of antibacterials (a mixture of neomycin, bacitracin, and pimaricin) reduces anxiety and increases exploratory behavior in the step-down and LDT tasks and hippocampal BDNF expression. However, treatment with amoxicillin has been reported to cause adverse neurological effects such as anxiety, hyperactivity, confusion, and behavioral changes as side effects in children. ${ }^{21}$

Therefore, to clarify these contradictions, we tried to isolate the commensal bacteria capable of causing anxiety-like behaviors. Of isolates, $K$. oxytoca significantly caused anxiety-like behaviors, suppressed BDNF expression in the hippocampus, and increased NF-kB activation in the hippocampus and colon. Furthermore, the intraperitoneal injection of LPS (isolated from $K$. oxytoca) also caused anxiety-like behaviors and suppressed BDNF expression in the hippocampus.

Furthermore, $K$. oxytoca and $\mathrm{KL}$ increased the recruits of microglia, monocytes, and DCs $\left(\mathrm{CD} 11 \mathrm{~b}^{+} \mathrm{CD} 45^{+}\right)$to the brain and the expression of IL-1R and IL-1 $\beta$ expression as well as the recruitment of macrophages and IL-1 expression, like ampicillin which are indicative of neuro-inflammation. These results suggest that ampicillin-proliferative $K$. oxytoca, which belongs to the $\gamma / \delta$ Proteobacteria, cause anxiety through suppressing BDNF expression and NF-KB activation in the brain through the induction of $\mathrm{Gl}$ inflammation.

Treatment with ampicillin or $K$. oxytoca significantly increased the population of Proteobacteria, whereas it decreased the abundance of the lactobacilli $L$. reuteri and $L$. johnsonii in the gut microbiota. We also found that $L$. reuteri isolated from the gut microbiota of healthy mice significantly attenuated ampicillin- or $K$. oxytoca-induced anxiety-like behaviors, increased BDNF expression, and suppressed NF-KB activation in the hippocampus. Furthermore, $L$. reuteri potently alleviated ampicillin- or $K$. oxytoca-induced colitis and restored gut microbiota composition and LPS levels. Davis et al. ${ }^{36}$ reported that lactobacilli, particularly $L$. reuteri, restored stress-induced disturbance of gut microbiota composition and attenuated anxiety. Bercik et al. ${ }^{45}$ reported that Bifidobacterium longum NCC3001 showed anxiolytic effects in rats. Messaoudi et al. ${ }^{46}$ also reported that a probiotic mixture composed of Lactobacillus helveticus R0052 and B. longum R0175 improved symptoms of anxiety and depression in volunteers. These results suggest that gut microbiota disturbance, particularly increased Proteobacteria population, and increase in LPS owing to diet, antibacterial treatment, and stresses may increase the occurrence of anxiety and colitis, which can change the secretion of neuroactive, endocrinal, and immunological molecules in brains and colons by increasing NF-KB activation and suppressing BDNF expression; these effects might be alleviated by correcting gut microbiota disturbance. Moreover, it is important to note that the failure of some antibacterials in inducing anxiety was previously reported $^{43}$ while this can be explained by the employment of different antibacterials, supporting that the interplay between antibacterials and anxiety could be antibacterial and microbiota specific.

In conclusion, ampicillin exposure can cause monocyte/macrophage-activated colitis by increasing the population of Proteobacteria including $K$. oxytoca, resulting in the occurrence of anxiety. These findings support the idea that the maintenance of favorable gut microbiota composition and the suppression of endotoxin productions by the increase of beneficial gut bacteria are beneficial to suppress and prevent the occurrence of anxiety through the amelioration of colitis.

\section{METHODS}

Culture of gut bacteria

For the culture of gut bacteria, the fresh feces of mice (approximately $0.1 \mathrm{~g}$ ) were suspended in the ninefold volume of GAM broth (Nissui Pharm. Co., Tokyo, Japan), diluted in a stepwise manner, and inoculated directly onto selective agar plates. ${ }^{47} \mathrm{DHL}$ agar (Nissui Pharm. Co.) plates were aerobically cultured for 1 day at $37^{\circ} \mathrm{C}$ and $\mathrm{BL}$ agar (Nissui Pharm. Co.) plates were anaerobically cultured for 3 days at $37^{\circ} \mathrm{C}$.

Lactobacilli ( $L$. reuteri, $L$. johnsonii, $L$. plantarum, and $L$. rhamnosus), K. oxytoca, E. coli, A. urinaeequi, and M. morganii isolated from mouse gut microbiota were cultured in GAM broth. Briefly, isolated bacteria were anaerobically cultured in $0.3 \mathrm{~L}$ of GAM broth at $37^{\circ} \mathrm{C}$ (an optical density at $600 \mathrm{~nm}, 0.6-0.8$ ), centrifuged at $5000 \times g$ for $20 \mathrm{~min}$, and washed with saline twice. Collected cells $\left(5 \times 10^{9} \mathrm{CFU} / \mathrm{mL}\right)$ were suspended in $1 \%$ glucose (for in vivo study). Isolated bacteria were identified by Gram staining, a sugar utilization test, and 16S rRNA sequencing.

\section{Animals}

Male C57BL/6 mice (age, 6 weeks old; weight, 20-22 g) supplied by Samtaco Animal Breeding Center (Seoul, Korea) were acclimated for 1 week before use. All animals were housed in wire cages at $24 \pm 2^{\circ} \mathrm{C}$ and $60 \pm 10 \%$ humidity with an alternating 12-12 h light/dark cycle. They were fed standard laboratory chow and tap water ad libitum. All animal experiments were approved by the Institutional Animal Care and Use Committee of the Kyung Hee University (IRB Number: KUASP(SE)-16011) and performed following the Kyung Hee University Guide for Laboratory Animals Care and Usage.

\section{Generation of mice with anxiety-like behaviors}

Mice with anxiety-like behaviors were generated by treatment with ampicillin (Sigma. St. Louis, MO) or fecal microbiota. Each group consisted of 8 mice (4 mice in each cage). First, mice were treated with ampicillin $(10,50$, and $100 \mathrm{mg} / \mathrm{kg} / \mathrm{day})$ via oral gavage once a day for 2 days, and anxiety-like behaviors were measured before ampicillin treatment and on the 4th and 10th days after ampicillin treatment (Supplement Figure S8A). Second, mice were orally gavaged with fecal microbiota of mice treated with ampicillin (FAP, $2.5 \mathrm{mg} / 0.1 \mathrm{~mL}$ ) once a day for 5 days, and anxiety-like behaviors were measured on the 5th day after treatment (Supplement Figure S8B). FAP suspension was prepared 
H-M Jang et al.

A
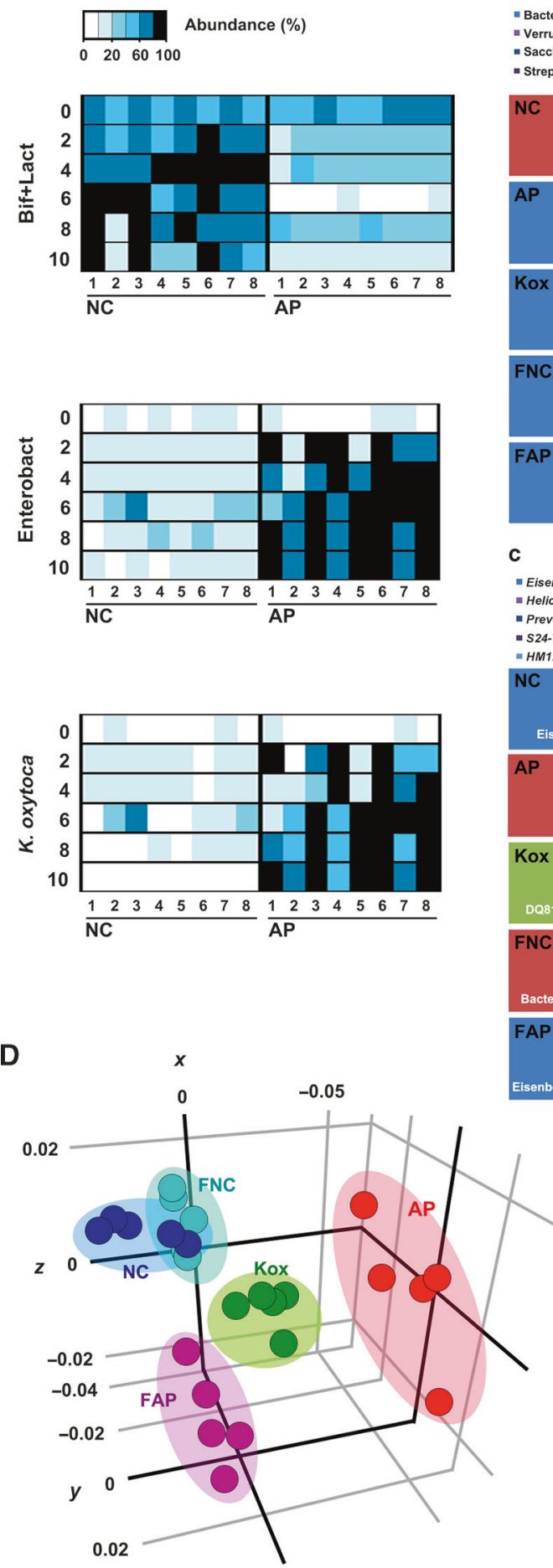
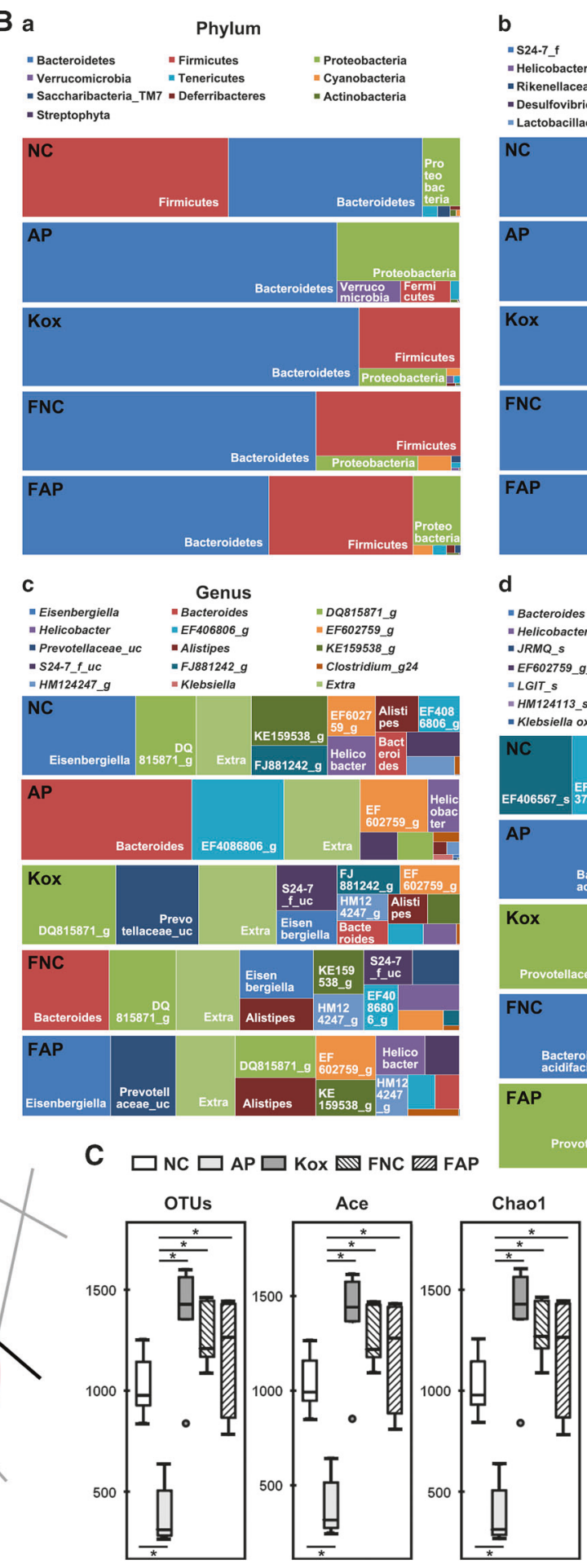

C
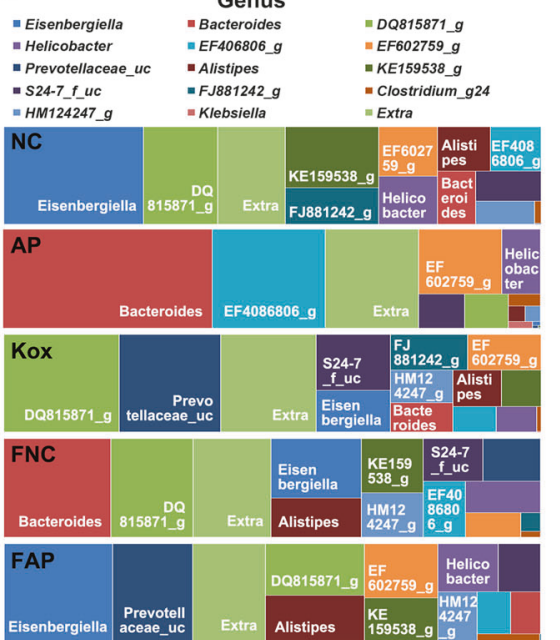

C $\square$ NC $\square$ AP $\square$ Kox FNC שFAP
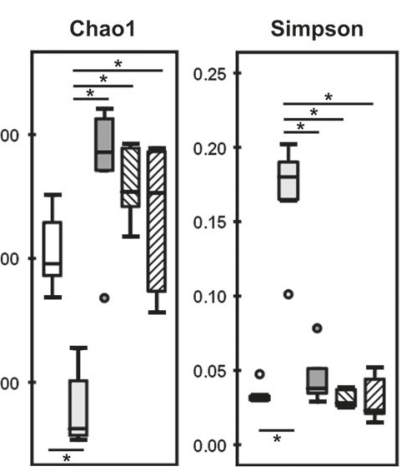

\section{d Species}

- Klebsiella oxytoca group Extra
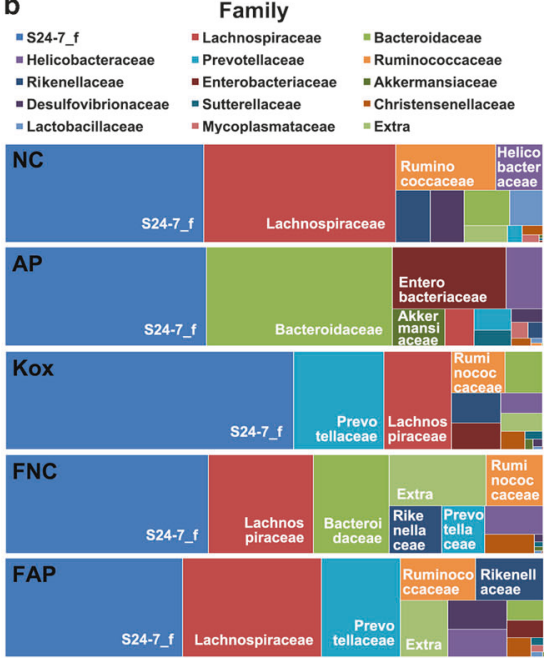

"Bacteroides acidifaciens "EF603904_s "Prevotellaceae_uc_s

- Helicobacter mesocricetorum "EF603701_s "S24-7_f_uc_s

- JRMQ_s "EF603109_s = Clostridium aldenense

-EF602759_g_uc E EF406567_s $=E$ EU505186_s

" LGIT_s "DQ815759_s "Bacteroides uniformis

$=$ HM124113_s "HM816924_s $=$ FJ881242_g_uc
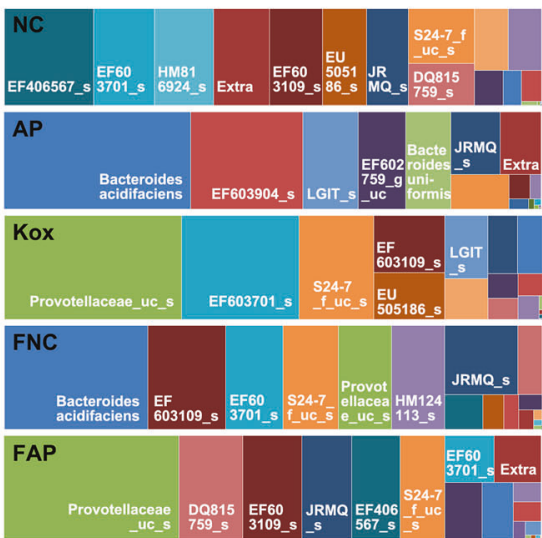

Fig. 3 Effects of orally administered ampicillin (AP), AP-treated mouse fecal microbiota of mice (FAP), normal control mouse fecal microbiota (FNC), and $K$. oxytoca on the fecal microbiota composition in mice. a Effects of ampicillin in the bifidobacteria, lactobacilli, Enterobacteriaceae, and $K$. oxytoca, assessed by the selective media (BL, selective for bifidobacteria (bif) and lactobacilli (lact); and DHL, selective for Enterobacteriaceae (Enterobact)) $(n=8)$. b Effects of ampicillin (AP) and K. oxytoca in the composition of gut microbiota using the pyrosequencing: (a) phylum, (b) family, (c) genus, and (d) species $(n=5)$. c Principal coordinate analysis (PCoA) plot. The plot shows the clustering pattern among normal control, AP-, and $K$. oxytoca-treated mice based on weighted pairwise Fast UniFrac analysis. $\mathbf{d}$ Effects on the number of sequences analyzed, operational taxonomic units (OTUs), estimated OTU richness (abundance-based coverage estimator (ACE) and Chao1). NC, AP, FNC, FAP, and Kox in figures indicate groups treated with vehicle alone (1\% dextrose), AP, FNC, FAP, and $K$. oxytoca, respectively. All data were expressed as mean \pm SD 
a

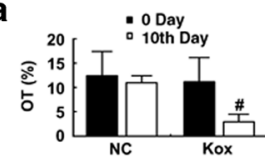

d

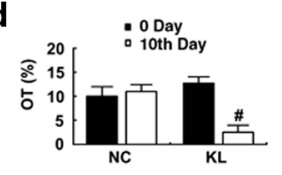

g

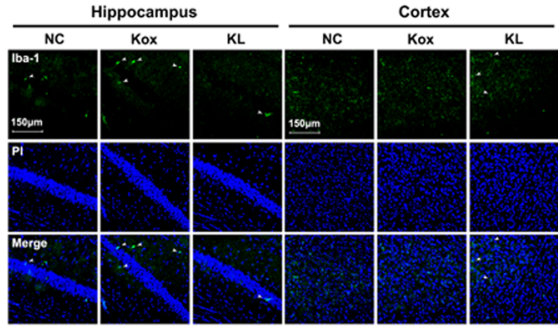

i

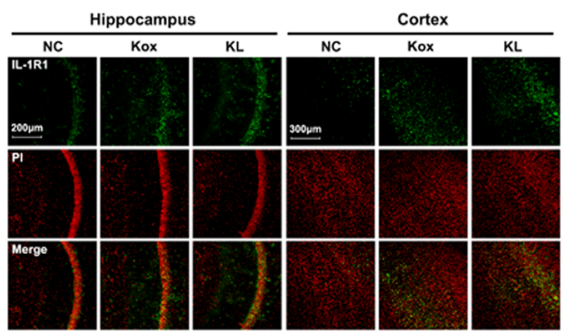

口NC.Hp 口Kox-Hp口NC.Ct
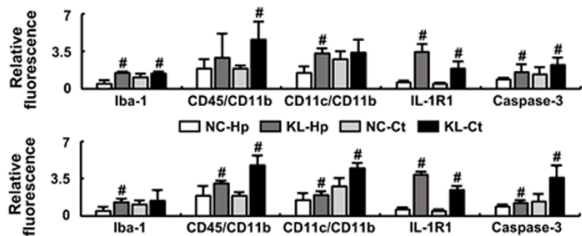

I

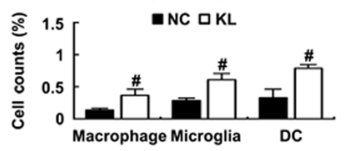

$\mathbf{m}_{\text {畐 }}$
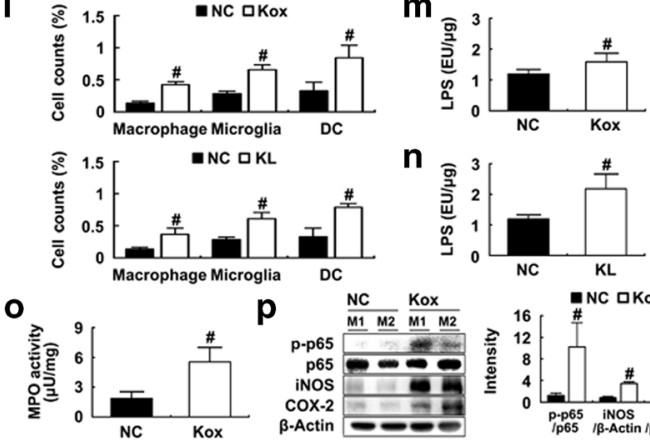

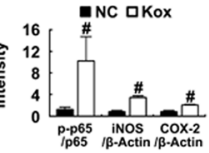

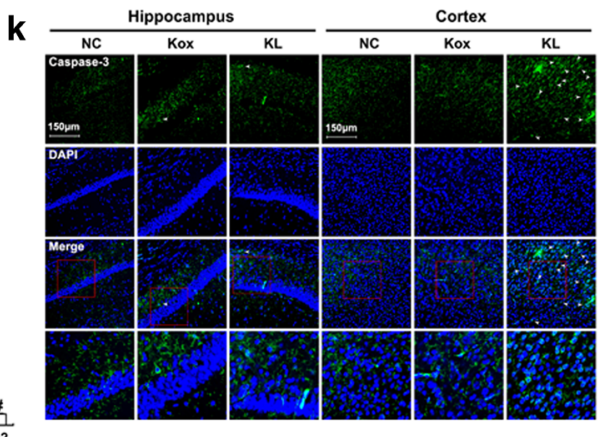

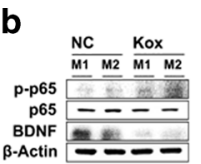

e

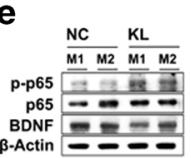

C

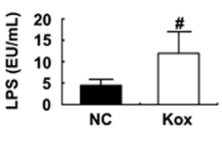

f

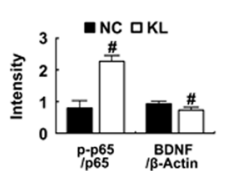

h

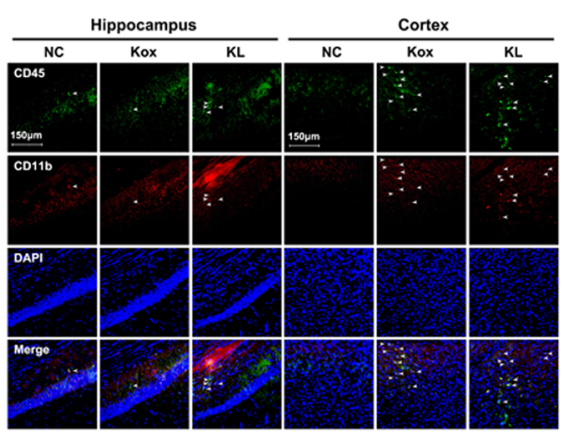

j

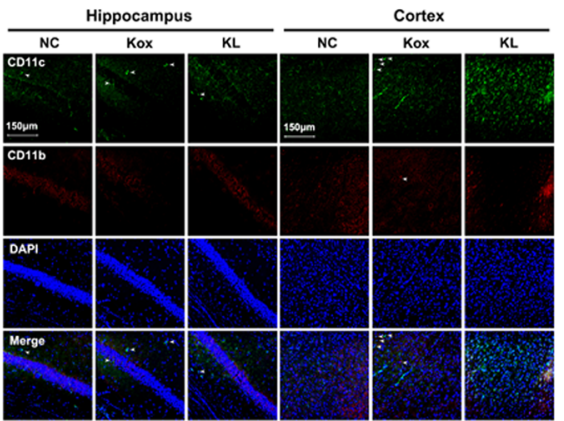

\section{t}

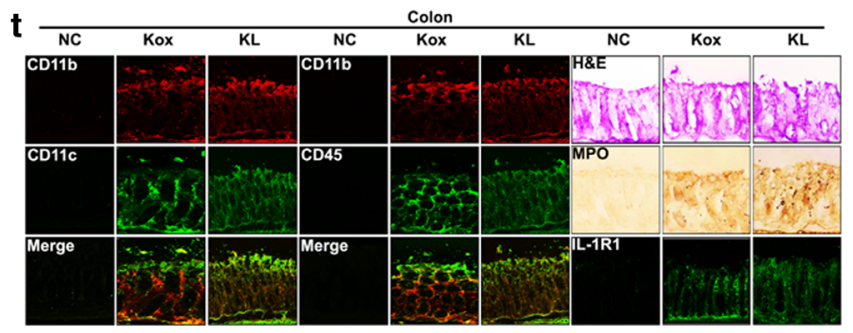

as follows: fresh feces were collected on the 10th day after the final ampicillin treatment, suspended in GAM broth on ice, centrifuged at $2000 \times g$ for $15 \mathrm{~min}$ at $4{ }^{\circ} \mathrm{C}$, washed with $1 \%$ dextrose, and used as the fecal microbiota $(25 \mathrm{mg} / \mathrm{mL}$ suspended in $1 \%$ dextrose). Third, mice were orally gavaged with $K$. oxytoca, $E$. coli, A. urinaeequi, or M. morganii $\left(1 \times 10^{9} \mathrm{CFU} /\right.$ mouse) once a day for 5 days, and anxiety-like behaviors were measured on the 5th day after treatment. Fourth, mice were intraperitoneally injected 
Fig. 4 Orally administered Klebsiella oxytoca or intraperitoneally injected $K$. oxytoca LPS (KL) induced anxiety-like behaviors and colitis in mice. Anxiety-like behaviors of $K$. oxytoca-treated mice were measured in the EPM (a). EPM were on 5th day after the final administration of $K$. oxytoca. Normal control mice (NC) were treated with vehicle alone. NF-KB (p-p65 and p65), BDNF, and $\beta$-actin were measured in the hippocampi by immunoblotting (b). Blood LPS levels (c), were analyzed by LAL kit. Anxiety-like behaviors of $K$. oxytoca lipopolysaccharide (KL)treated mice were measured in the EPM (d). EPM were on 5th day after the final administration of KL. NF-kB (p-p65 and p65), BDNF, and $\beta$-actin in the hippocampi (e) and blood LPS levels (f) were analyzed by immunoblotting and LAL kit, respectively. Microglia (g), monocytes (h), IL-1Rexpressing (i), dendritic (j), and apoptotic neuron cells (k) in the brain were assayed by a confocal microscope. Microglia, monocytes, and dendritic cells were also assayed by a flow cytometer (I). Brain Fecal LPS levels of mice treated with K. oxytoca (m) or KL (n) were measured by LAL assay kit. Colitis markers myeloperoxidase activity (o), and iNOS, COX-2, NF-KB (p), and tight junction proteins (occludin and claudin-1), and $\beta$-actin (o), were assessed by immunoblotting. FITC-dextran orally administered in $K$. oxytoca-treated mice was absorbed into the blood (r). Fecal LPS levels of mice treated with $K$. oxytoca were measured by LAL assay kit (s). Macrophages, dendritic, and IL-1R1-expressing cells recruited/activated into the colon were assayed by a confocal microscope (t). NC, Kox, and KL in the $x$-axis of figures indicate groups treated with vehicle alone ( $1 \%$ dextrose), K. oxytoca, or KL, respectively. All data were expressed as mean \pm SD $(n=8)$; ${ }^{*} p<0.05$ vs. normal control (NC) group

with LPS, which was isolated from $K$. oxytoca according to the method of Jang et al., ${ }^{19}$ or $L$. reuteri exopolysaccharide (LE), which was isolated from $L$. reuteri according to the method of Yun et al., ${ }^{48}$ once a day for 5 days and the anxiety-like behaviors were measured on the 5th day after the final treatment with LPS (Supplement Figure S8C).

To evaluate the anxiolytic-like effects of lactobacilli, mice were randomly divided into normal control and anxiety-induced groups with ampicillin, or fecal microbiota of mice treated with ampicillin, K. oxytoca, and KL (LPS isolated from K. oxytoca) according to the method of Jang et al. ${ }^{19}$ Mice were then treated with probiotic $L$. reuteri, $L$. johnsonii, L. plantarum, L. rhamnosus $\left(2 \times 10^{8}, 1 \times 10^{9}\right.$, or $5 \times 10^{9}$ CFU/mouse, p.o.), or vehicle ( $1 \%$ dextrose). Mice were treated with lactobacilli once a day for 5 days from next day after the treatment with anxiety inducers and killed $28 \mathrm{~h}$ after the final treatment with lactobacilli.

Behavioral tasks to text for anxiety

The EPM task was performed in the plus-maze apparatus (consisting of two open $(30 \times 7 \mathrm{~cm})$ and two enclosed arms $(30 \times 7 \mathrm{~cm})$ with $20 \mathrm{~cm}$-high walls extending from a central platform $(7 \times 7 \mathrm{~cm})$ on a single central support to a height of 60 $\mathrm{cm}$ above the floor) for 5 min according to the method of Oh et al. $^{49}$ The LDT task was performed in the light/dark box apparatus $(45 \times 25 \times 25 \mathrm{~cm}$, consisting of two chambers made of black and white polywoods (walls) and Plexiglass (floor) connected by an opening $(7.5 \times 7.5 \mathrm{~cm})$ located at floor level in the center of the dividing wall) for $5 \mathrm{~min}$ according to the method of Jindal et al. ${ }^{50}$ The MB task was performed in a cage $(30 \times 36 \times 13 \mathrm{~cm}$, constructed of smooth, opaque plastic with a $5-\mathrm{cm}$ layer of sawdust) for $30 \mathrm{~min}$ as described by Savignac et al. ${ }^{37}$

\section{Immunoblotting}

The colon and hippocampal tissues were lysed with ice-cold lysis RIPA buffer containing $50 \mathrm{mM}$ Tris- $\mathrm{HCl}(\mathrm{pH} 8.0), 150 \mathrm{mM}$ sodium chloride, $1.0 \%$ Igepal CA-630 (NP-40), $0.5 \%$ sodium deoxycholate, $0.1 \%$ sodium dodecyl sulfate, $1 \%$ phosphatase inhibitor cocktail and a protease inhibitor cocktail and were centrifuged at $4{ }^{\circ} \mathrm{C}$ and $10,000 \times g$ for $10 \mathrm{~min} .{ }^{19}$ The supernatants were electrophoresed by sodium dodecyl sulfate-polyacrylamide gel electrophoresis and transferred to a nylon membrane, blocked with 5\% nonfat dried-milk proteins, probed with the corresponding antibodies (Cell Signaling, Beverly, MA), and washed with phosphatebuffered saline with tween-20. Proteins were detected with horseradish peroxidase-conjugated secondary antibodies and their bands were visualized with the enhanced chemiluminescence detection.

Enzyme-linked immunosorbent assay (ELISA)

For the assay of blood corticosterone and cytokines, mice were killed $2 \mathrm{~h}$ after the EPM task and bloods were collected from carotid artery. ${ }^{19,47}$ Bloods were centrifuged at $3000 \times g$ for 5 min at $4{ }^{\circ} \mathrm{C}$ and sera were prepared. Levels of corticosterone, IL-6, and TNF- $a$ in the sera and cytokines in the supernatant of colon homogenates were measured using commercial ELISA kits (Ebioscience, San Diego, CA).

\section{Quantitative polymerase chain reaction (qPCR)}

Real-time PCR for Firmicutes, $\beta$-Proteobacteria, $\delta / \gamma$-Proteobacteria, $\varepsilon$-Proteobacteria, TM7, Verrucomicrobia, Actinobacteria, and Bacteroidetes was performed with $100 \mathrm{ng}$ total DNA isolated from the colon fluid with SYBER premix in a Takara thermal cycler. ${ }^{51}$ The thermal cycling conditions were as follows: $95^{\circ} \mathrm{C}$ for $30 \mathrm{~s}$, followed by 35 cycles of denaturation and amplification at $95^{\circ} \mathrm{C}$ for $5 \mathrm{~s}$, and $630^{\circ} \mathrm{C}$ for $30 \mathrm{~s}$, respectively. Bacterial levels were calculated relative to bacterial rRNA, using Microsoft Excel. The primers were used in supplement Table S1.

\section{Immunofluorescence assay}

Immunostaining analysis of brain and colon slices was performed according to the method of Tronnes et al. ${ }^{52}$ Monocytes were stained with antibodies with CD11b (1:150, Abcam) and CD45 (1:100, Abcam) and DCs were stained with antibodies against CD11b (1:150, Abcam) and CD11c (1:150, Abcam). IL-1R-expressing cells were stained with an antibody against IL-1R (1:200, Abcam). Microglial cells were visualized by staining with an lba- 1 antibody (1:100, Santa Cruz Biotechnology). Briefly, the colon and brain tissues were cryoprotected in $30 \%$ sucrose-PBS and then frozen with optimal cutting temperature compound and stored at $-80^{\circ} \mathrm{C}$ until processed. These tissue blocks were cryosectioned through the coronal plane at a thickness of $30 \mu \mathrm{m}$, stored at $4{ }^{\circ} \mathrm{C}$ in the storing solution (30\% glycerol, $30 \%$ ethylene glycol in PBS), permeabilized in $0.5 \%$ Triton X-100 for $5 \mathrm{~min}$, and blocked in $10 \%$ normal serum with PBS with tween-20 for $30 \mathrm{~min}$ in a free floating condition. The sections were incubated for $16 \mathrm{~h}$ at $4{ }^{\circ} \mathrm{C}$ with antibodies. Secondary antibodies conjugated with Alexa Fluor 488 (1:1000, Invitrogen) or Alexa Fluor 594 (1:500, Abcam) were used. Nuclei were stained with 4',6-diamidino-2-phenylindole, dilactate (DAPI, Sigma, St. Louis, MO) or counterstained with $1 \mu \mathrm{g} / \mathrm{mL}$ propidium iodide (PI, Sigma) for $15 \mathrm{~min}$. Apoptotic neuron cells were stained using the NeuN and caspase- 3 antibodies according to the method of Sabri et al. ${ }^{53}$ Immunostained sections were scanned with a confocal laser microscope (LSM510, Carl Zeiss, Oberkochen, Germany).

\section{Flow cytometry}

To assay the populations of microglia, DCs, and monocytes in the brain, mouse brains were cut into small pieces, kept with EDTA/ HEPES/PBS on ice, minced, and digested for $1 \mathrm{~h}$ with $0.5 \mathrm{mg} / \mathrm{mL}$ collagenase type IV, DNase I $20 \mathrm{U} / \mathrm{mL}$ at $37^{\circ} \mathrm{C}$. The cells were filtered, separated by percoll, fixed, and stained with anti-CD11b, CD11C, and/or CD45 antibodies. 
A a
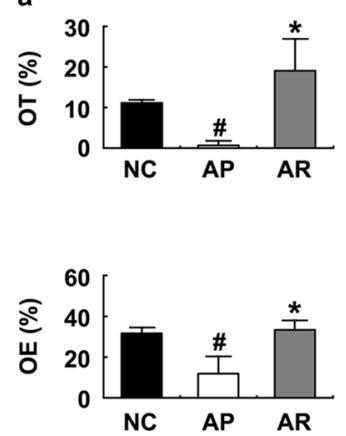

B
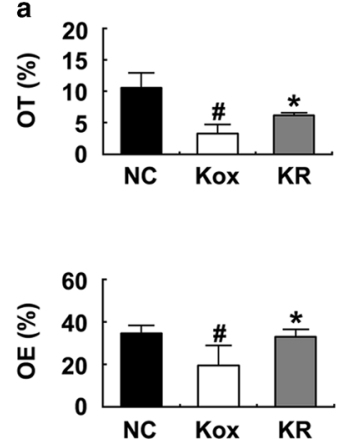

b
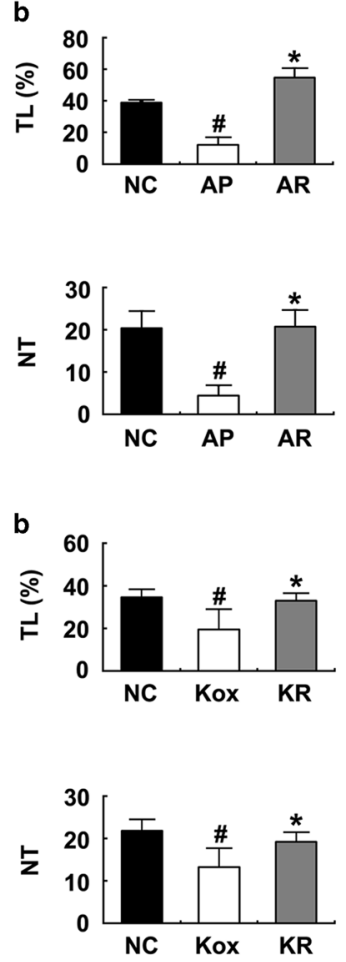

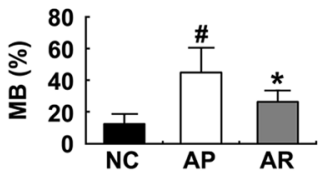

d
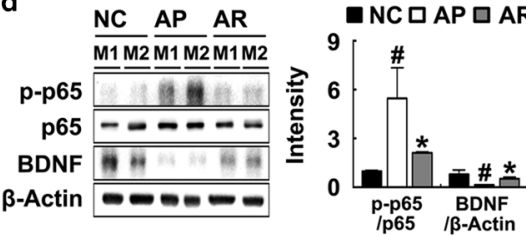

c

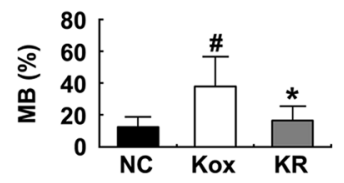

d

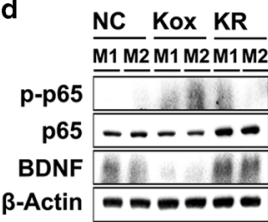

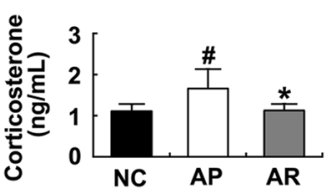

f

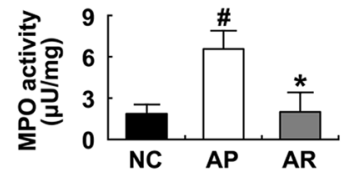

e
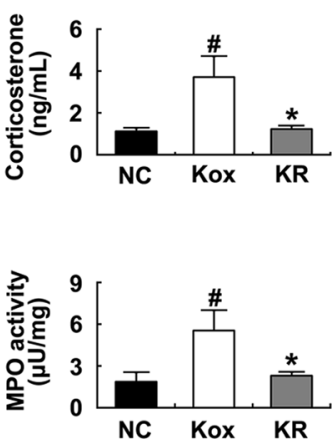

Fig. 5 Oral administration of Lactobacillus reuteri alleviated immobilization stress ampicillin (AP)- and Klebsiella oxytoca-induced anxiety-like behaviors and colitis in mice. a Effect of $L$. reuteri on the occurrence of anxiety and colitis in mice treated with AP. An AL group was orally treated with $L$. reuteri in mice pretreated with AP and anxiety-like behaviors of mice were measured in the EPM (a), LDT (b), and MB tests (c). Normal control mice (NC) were not treated with AP and L. reuteri. BDNF, NF- $\mathrm{KB}$, and $\beta$-actin were measured in the hippocampus by immunoblotting (d). Blood corticosterone (e) was analyzed by ELISA or LAL kit. Myeloperoxidase activity (f) was measured in the colon. $\mathbf{b}$ Effect of $L$. reuteri on the occurrence of anxiety and colitis in mice treated with $K$. oxytoca. An AL group was orally treated with $L$. reuteri in mice pretreated with $K$. oxytoca and anxiety-like behaviors of mice were measured in the EPM (a), LDT (b), and MB tests (c). Normal control mice (NC) were not treated with $A P$ and $L$. reuteri. BDNF, NF-KB, and $\beta$-actin were measured in the hippocampi by immunoblotting (d). Blood corticosterone (e) was analyzed by ELISA or LAL kit. Myeloperoxidase activity (f) was measured in the colon. NC, AP, and AR in the $x$-axis were treated with vehicle (saline) alone, AP, and AP plus L. reuteri, respectively. NC, Kox, and KR in the $x$-axis were treated with vehicle (1\% dextrose), $K$. oxytoca, and K. oxytoca plus $L$. reuteri, respectively. All data were expressed as the mean \pm SD $(n=8) ;{ }^{*} p<0.05$ vs. normal control (NC) group; ${ }^{*} p<0.05$ vs. AP or K. oxytoca-treated group

\section{Pyrosequencing}

Genomic DNA was extracted from the fresh stools of mice using a commercial DNA isolation kit (QIAamp DNA stool mini kit), as previously reported. ${ }^{19,47}$ Amplification of the genomic DNA was performed using barcoded primers, which targeted the V3 to V4 region of the bacterial $16 \mathrm{~S}$ rRNA gene. Pyrosequencing was performed using a 454 GS FLX Titanium Sequencing System (Roche, Branford, CT). Sequence reads were identified using the EzTaxon-e database (http://eztaxon-e.ezbiocloud.net/) on the basis of 16S rRNA sequence data. The number of sequences analyzed, observed diversity richness (OTUs), estimated OTU richness (ACE and Chao1), and coverage indicated in Supplement Table S2 indicated in Table S1 were calculated using the Mothur program and defined considering a cut-off value of $97 \%$ similarity with the 16S rRNA gene sequences. In all, 454 pyrosequencing reads have been deposited in the $\mathrm{NCBI}$ (National Center for Biotechnology Information) short read archive under accession number SRP116671.

Limulus amoebocyte lysate ( $L A L)$ assay

Fecal and blood endotoxin contents were determined using the diazo-coupled LAL assay kit (Cape Cod Inc., East Falmouth, MA). ${ }^{19}$, 47 Briefly, for the determination of fecal endotoxin concentration, feces $(20 \mathrm{mg})$ were collected in $30 \mathrm{~mL}$ of PBS in a pyrogen-free tube and sonicated for $1 \mathrm{~h}$ on ice. After centrifugation at $400 \times g$ for $15 \mathrm{~min}$, the upper $20 \mathrm{~mL}$ was collected, sterilized by filtration through a $0.45 \mu \mathrm{m}$ filter, followed by re-filtration through a 0.22 $\mu \mathrm{m}$ filter, and inactivated for $10 \mathrm{~min}$ at $70^{\circ} \mathrm{C}$. The endotoxin content of the filtrate was assayed according to the manufacturer's protocol.

For the determination of blood endotoxin concentration, plasma was diluted in pyrogen-free water 10 -fold, inactivated at $70^{\circ} \mathrm{C}$ for $10 \mathrm{~min}$, and then centrifuged $(3000 \times g, 10 \mathrm{~min})$. The endotoxin content of the supernatant was assayed.

In vivo intestinal permeability assay of FITC-dextran Mice treated with or without ampicillin, FAP, FNC, or $K$. oxytoca were orally dosed with $0.1 \mathrm{~mL}$ of FITC-dextran $(200 \mu \mathrm{g} / \mathrm{kg})$. Normal control mice were dosed with a corresponding dose of PBS. Exactly $2 \mathrm{~h}$ and $4 \mathrm{~h}$ after the administration of FITC-dextran, the blood was collected from the tail. Blood was centrifuged for 10 min $\left(1500 \times g\right.$ and $\left.4^{\circ} \mathrm{C}\right)$ and the supernatant was mixed with the same volume of PBS, and the fluorescence was measured in black 96-well microtiter plates (Proxiplate-96 F, Perkin Elmer, Waltham, MA, USA) using a FLOUstar Omega (BMG, LABTECH, Ortenberg, Germany) with excitation at $495 \mathrm{~nm}$ and emission at $540 \mathrm{~nm}$.

Statistical analysis

Data are indicated as mean \pm SD. Significant differences were analyzed using one-way analysis of variance followed by Duncan's multiple range test $(P<0.05)$. All statistical analyses were performed using GraphPad Prism 5.0 (Graphpad, La Jolla, CA) (Supplement Table S2). 


\section{ACKNOWLEDGEMENTS}

This research was also supported by the Basic Science Research Program through the National Research Foundation of Korea (NRF), funded by the Ministry of Science and ICT (NRF- 2017R1A5A2014768).

\section{AUTHOR CONTRIBUTIONS}

D.-H.K and M.J.H. conceived the idea for this study. H.-M.J., H.-J.L., M.J.H., and D.-H.K. designed the experiment. H.-M.J., H.-J.L., and S.-E.J. performed the experiment. H.-M. J., H.-J.L., and D.-H.K. analyzed the data. H.-M.J. and D.-H.K. wrote the main manuscript text. All authors reviewed and approved the contents of the manuscript.

\section{ADDITIONAL INFORMATION}

The online version of this article (https://doi.org/10.1038/s41385-018-0042-3) contains supplementary material, which is available to authorized users.

Competing interests: The authors declare no competing interests.

Publisher's note: Springer Nature remains neutral with regard to jurisdictional claims in published maps and institutional affiliations.

\section{REFERENCES}

1. Hart, P. C. et al. Experimental models of anxiety for drug discovery and brain research. Methods Mol. Biol. 1438, 271-91 (2015).

2. Baxter, A. J. et al. Challenging the myth of an "epidemic" of common mental disorders: trends in the global prevalence of anxiety and depression between 1990 and 2010. Depress. Anxiety 31, 506-16 (2014).

3. Eisenberg, D. M. et al. Trends in alternative medicine use in the United States, 1990-1997: results of a follow-up national survey. JAMA 280, 1569-75 (1998)

4. Langgartner, D. et al. Individual differences in stress vulnerability: the role of gut pathobionts in stress-induced colitis. Brain Behav. Immun. 64, 23-32 (2017).

5. Padgett, D. A.. \& Glaser, R. How stress influences the immune response. Trends Immunol. 24, 444-8 (2003).

6. Amitai, M. et al. The relationship between plasma cytokine levels and response to selective serotonin reuptake inhibitor treatment in children and adolescents with depression and/or anxiety disorders. J. Child Adolesc. Psychopharmacol. 26, 727-32 (2016)

7. Fan, J. M., Chen, X. Q. \& Du, J. Z. Prenatal stress, anxiety and depression: a mechanism involving CRH peptide family. Neuroendocrinol. Lett. 35, 429-39 (2014).

8. Haddad, J. J., Saade, N. E. \& Safieh-Garabedian, B. Cytokines and neuro-immuneendocrine interactions: a role for the hypothalamic-pituitary-adrenal revolving axis. J. Neuroimmunol. 133, 1-19 (2002).

9. Sekiyama, A. et al. A role of the adrenal gland in stress-induced up-regulation of cytokines in plasma. J. Neuroimmunol. 171, 38-44 (2006).

10. Foster, J. A. Gut microbiome and behavior: focus on neuroimmune interactions. Int. Rev. Neurobiol. 131, 49-65 (2016).

11. Kelly, J. R. et al. Transferring the blues: depression-associated gut microbiota induces neurobehavioural changes in the rat. J. Psychiatr. Res. 82, 109-18 (2016).

12. Statovci, D., Aguilera, M., MacSharry, J. \& Melgar, S. The impact of western diet and nutrients on the microbiota and immune response at mucosal tnterfaces. Front. Immunol. 8, 838 (2017).

13. Sharon, G., Sampson, T. R., Geschwind, D. H. \& Mazmanian, S. K. The central nervous system and the gut microbiome. Cell 167, 915-32 (2016).

14. Carabotti, M., Scirocco, A., Maselli, M. A. \& Severi, C. The gut-brain axis: interactions between enteric microbiota, central and enteric nervous systems. Ann. Gastroenterol. 28, 203-9 (2015).

15. Mackos, A. R., Varaljay, V. A., Maltz, R., Gur, T. L. \& Bailey, M. T. Role of the intestinal microbiota in host responses to stressor exposure. Int. Rev. Neurobiol. 131, 1-19 (2016).

16. Gareau, M. G. et al. Bacterial infection causes stress-induced memory dysfunction in mice. Gut 60, 307-17 (2011)

17. Liu, X., Cao, S. \& Zhang, X. Modulation of gut microbiota-brain axis by probiotics, prebiotics, and diet. J. Agric. Food Chem. 63, 7885-95 (2015).

18. Yang, B. G., Hur, K. Y. \& Lee, M. S. Alterations in gut microbiota and immunity by dietary fat. Yonsei Med. J. 58, 1083-91 (2017).

19. Jang, S. E. et al. Gastrointestinal inflammation by gut microbiota disturbance induces memory impairment in mice. Mucosal Immunol. 11, 369-79 (2017).

20. Cole, T. S., Clark, M. E., Jenkins, A. J. \& Clark, J. E. Pediatric focal intracranial suppuration: a UK single-center experience. Childs Nerv. Syst. 28, 2109-14 (2012).

21. Atli, O. et al. Evidence for neurotoxicity associated with amoxicillin in juvenile rats. Hum. Exp. Toxicol. 35, 866-676 (2006).
22. Leclercq, S. et al. Low-dose penicillin in early life induces long-term changes in murine gut microbiota, brain cytokines and behavior. Nat. Commun. 8, 15062 (2017).

23. Bercik, P. \& Collins, S. M. The effects of inflammation, infection and antibiotics on the microbiota-gut-brain axis. Adv. Exp. Med. Biol. 817, 279-89 (2014).

24. Lurie, I., Yang, Y. X., Haynes, K., Mamtani, R. \& Boursi, B. Antibiotic exposure and the risk for depression, anxiety, or psychosis: a nested case-control study. J. Clin. Psychiatry 76, 1522-28 (2015).

25. Kerman, D. H. \& Deshpande, A. R. Gut microbiota and inflammatory bowel disease: the role of antibiotics in disease management. Postgrad. Med. 126, 7-19 (2014).

26. Barbara, G. et al. Interactions between commensal bacteria and gut sensorimotor function in health and disease. Am. J. Gastroenterol. 100, 2560-8 (2005).

27. Stilling, R. M., Dinan, T. G. \& Cryan, J. F. Microbial genes, brain and behaviour epigenetic regulation of the gut-brain axis. Genes Brain Behav. 13, 69-86 (2014).

28. Clarke, G. et al. The microbiome-gut-brain axis during early life regulates the hippocampal serotonergic system in a sex-dependent manner. Mol. Psychiatry 18, 666-73 (2013).

29. Diaz Heijtz, R. et al. Normal gut microbiota modulates brain development and behavior. Proc. Natl. Acad. Sci. USA 108, 3047-52 (2011).

30. Wostmann, E. \& Bruckner-Kardoss, E. Development of cecal distention in germfree baby rats. Am. J. Physiol. 197, 1345-46 (1959).

31. Sudo, N. et al. Postnatal microbial colonization programs the hypothalamicpituitary-adrenal system for stress response in mice. J. Physiol. 558, 263-75 (2004).

32. Tannock, G. W. \& Savage, D. C. Influences of dietary and environmental stress on microbial-populations in murine gastrointestinal-tract. Infect. Immun. 9, 591-8 (1974).

33. Bailey, M. T. et al. Stressor exposure disrupts commensal microbial populations in the intestines and leads to increased colonization by Citrobacter rodentium. Infect. Immun. 78, 1509-19 (2010).

34. Bailey, M. T. et al. Exposure to a social stressor alters the structure of the intestinal microbiota: Implications for stressor-induced immunomodulation. Brain Behav. Immun. 25, 397-407 (2011).

35. Marin, I. A. et al. Microbiota alteration is associated with the development of stress-induced despair behavior. Sci. Rep. 7, 43859 (2017).

36. Davis, D. J. et al. Lactobacillus plantarum attenuates anxiety-related behavior and protects against stress-induced dysbiosis in adult zebrafish. Sci. Rep. 6, 33726 (2016).

37. Savignac, H. M., Kiely, B., Dinan, T. G. \& Cryan, J. F. Bifidobacteria exert strainspecific effects on stress-related behavior and physiology in BALB/c mice. Neurogastroenterol. Motil. 26, 1615-27 (2014).

38. Foster, J. A. \& McVey Neufeld, K. A. Gut-brain axis: how the microbiome influences anxiety and depression. Trends Neurosci. 36, 305-12 (2013).

39. Rieder, R., Wisniewski, P. J., Alderman, B. L. \& Campbell, S. C. Microbes and mental health: a review. Brain Behav. Immun. 66, 9-17 (2017).

40. Bercik, P. et al. The intestinal microbiota affect central levels of brain-derived neurotropic factor and behavior in mice. Gastroenterology 141, 599-609 (2011).

41. Desbonnet, L. et al. Gut microbiota depletion from early adolescence in mice: Implications for brain and behaviour. Brain Behav. Immun. 48, 165-73 (2015).

42. Fröhlich, E.E. et al. Cognitive impairment by antibiotic-induced gut dysbiosis: Analysis of gut microbiota-brain communication. Brain Behav. Immun. 56, 140-55 (2016).

43. Hoban, A. E. et al. Behavioural and neurochemical consequences of chronic gut microbiota depletion during adulthood in the rat. Neuroscience 339, 463-77 (2016).

44. Vogt, M. A. et al. Minocycline does not evoke anxiolytic and antidepressant-like effects in C57BL/6 mice. Behav. Brain Res. 301, 96-101 (2016).

45. Bercik, P. et al. The anxiolytic effect of Bifidobacterium longum NCC3001 involves vagal pathways for gut-brain communication. Neurogastroenterol. Motil. 23, 1132-9 (2011)

46. Messaoudi, M. et al. Assessment of psychotropic-like properties of a probiotic formulation (Lactobacillus helveticus R0052 and Bifidobacterium longum R0175) in rats and human subjects. Br. J. Nutr. 105, 755-64 (2011).

47. Kim, K. A., Gu, W., Lee, I. A., Joh, E. H. \& Kim, D. H. High fat diet-induced gut microbiota exacerbates inflammation and obesity in mice via the TLR4 signaling pathway. PLoS One 7, e47713 (2012).

48. Yun et al. Isolation and characterization of exopolysaccharide producing lactic acid bacteria from Korean soy sauce and soybean paste. Korean J. Microbiol. Biotechnol. 41, 190-7 (2013).

49. Oh, H. A., Kim, D. E., Choi, H. J., Kim, N. J. \& Kim, D. H. Anti-stress effects of 20(S)protopanaxadiol and 20(S)-protopanaxatriol in immobilized mice. Biol. Pharm. Bull. 38, 331-5 (2015) 
Evidence for interplay among antibacterial-induced gut... H-M Jang et al.

50. Jindal, A., Mahesh, R. \& Kumar, B. Anxiolytic-like effect of linezolid in experimental mouse models of anxiety. Prog. Neuropsychopharmacol. Biol. Psychiatry 40, 47-53 (2013).

51. Yang, Y. W. et al. Use of $16 \mathrm{~S}$ rRNA gene-targeted group-specific primers for realtime PCR analysis of predominant bacteria in mouse feces. Appl. Environ. Microbiol. 81, 6749-56 (2015).
52. Tronnes, A. A. et al. Effects of lipopolysaccharide and progesterone exposures on embryonic cerebral cortex development in mice. Reprod. Sci. 23, 771-8 (2016).

53. Sabri, M, Kawashima, A., Ai, J. \& Macdonald, R.L. Neuronal and astrocytic apoptosis after subarachnoid hemorrhage: a possible cause for poor prognosis. Brain Res. 1238, 163-71 (2008). 